\title{
Translation Invariant Equilibrium States of Ferromagnetic Abelian Lattice Systems
}

\author{
Charles-Edouard Pfister \\ Département de Mathématiques, Ecole Polytechnique Fédérale, 61, Av. de Cour, CH-1007 Lausanne, \\ Suisse
}

\begin{abstract}
The structure of the set of all translation invariant equilibrium states is determined for all temperatures, for which the free energy is differentiable. Models with several phase transitions are discussed rigorously.
\end{abstract}

\section{Introduction}

The class of ferromagnetic models considered in this paper includes the Ising model, $X Y$ or Rotator model, Ashkin-Teller model, $\mathbb{Z}_{n}$-model, Potts model and so on. For any temperature one can construct a translation invariant equilibrium state, $\langle\cdot\rangle^{0}$, which is also an extremal equilibrium state (see Sect. 3). Moreover, if $S_{0}$ is the internal symmetry group (see Sect. 4) and $S(\beta)$ the subgroup of $S_{0}$, which leaves $\langle\cdot\rangle^{0}$ invariant, then all equilibrium states are $S(\beta)$-invariant. In particular, when $S_{0}=S(\beta)$, there is no symmetry breakdown of $S_{0}$. On the other hand, when $S\left(\beta_{0}\right) \neq S_{0}$, all equilibrium states are $S\left(\beta_{0}\right)$-invariant and therefore there is a natural action of the quotient group $S_{0} / S\left(\beta_{0}\right)$ on the equilibrium states. Using this action on $\langle\cdot\rangle^{0}$, one obtains new extremal equilibrium states $\langle\cdot\rangle^{\theta}, \theta \in S_{0} / S\left(\beta_{0}\right)$. Let $\lambda$ be any probability measure on $S_{0} / S\left(\beta_{0}\right)$, which is translation invariant (with respect to the action of $\mathbb{Z}^{d}$ on $S_{0} / S\left(\beta_{0}\right)$ ). The state

$$
\int_{S_{0} / S\left(\beta_{0}\right)} \lambda(d \theta)\langle\cdot\rangle^{\theta}
$$

is clearly a translation invariant equilibrium state. The main result of Sect. 4 ensures, that all translation invariant equilibrium states at inverse temperature $\beta_{0}$ are given by (1.1), if and only if the free energy is differentiable with respect to $\beta$ at $\beta_{0}$. This result was already known for Ising ferromagnetic systems by the works of Slawny [1] Lebowitz [2], [3], and Bricmont, Lebowitz, Pfister [4]. The main technical tool is correlation inequalities [5], which are derived using ideas of Ginibre in his basic paper on correlation inequalities [6]. One obtains in this way results similar to those of Lebowitz in [2]. 
In the second part of the paper (Sect. 5), one constructs simple models with several phase transitions with symmetry breakdown. One can also consider gauge models with several phase transitions associated with different Wilson loops. The main tool is Ginibre inequalities [6]. This second part is almost independent from the first part, except for notations, which are fixed in Sect. 2.

\section{Notations}

All models are defined on the lattice $\mathbb{Z}^{d}$, and the state space at $x \in \mathbb{Z}^{d}$ is the same for all $x$. It is either the compact abelian group $T=\mathbb{R} / 2 \pi \mathbb{Z}$, or the discrete subgroup of $T, U_{p}=\left\{\frac{k \cdot 2 \pi}{p}, k=0,1, \ldots, p-1\right\}$. The state space is always considered as a measure space with the normalized Haar measure. The configuration space $G$ is the set of functions on $\mathbb{Z}^{d}$ with values in the state space. Here $G$ is an abelian compact group with the addition defined pointwise and with product topology. Its elements are denoted by $\theta, \varphi, \theta_{i}$ etc. Also $G$ is a measure space (with the product structure) and the normalized Haar measure is denoted by $d \theta$. The configuration space has the following important property : given any subset $\Lambda \subset \mathbb{Z}^{d}$, the projection $\pi_{\Lambda}$ assigns to each $\theta \in G$ its restriction $\theta_{\Lambda}$ on the set $\Lambda$. Let $G(\Lambda)=\pi_{\Lambda} G$ and $\bar{\Lambda}=\mathbb{Z}^{d} \backslash \Lambda$. Then

$$
G=G(\Lambda) \times G(\bar{\Lambda}), \quad d \theta=d \theta_{\Lambda} \otimes d \theta_{\bar{\Lambda}},
$$

where $d \theta_{\Lambda}=\pi_{\Lambda} d \theta$.

Since $G$ is a compact abelian group, it is natural to consider the dual of $G$, which is isomorphic to the set $\Gamma$ of all functions on $\mathbb{Z}^{d}$ with finite support and values in $\mathbb{Z}$, if the state space is $T$, otherwise in $\mathbb{Z}_{p}$. Its elements are denoted by $m, n, m_{i}$ etc. By convention

$$
m \theta=\sum_{x} m(x) \theta(x)
$$

There is a natural action of $\mathbb{Z}^{d}$ on $G$, respectively $\Gamma$. For example, the translate of $m$ by $y \in \mathbb{Z}^{d}$ is the function $m_{y}$,

$$
m_{y}(x)=m(x-y) .
$$

For the sake of simplicity all interactions have finite range and are translation invariant. Hence an interaction is defined by $k$ local observables. The next restriction on the choice of these local observables is of course essential for this paper: All local observables are of the type

$$
-J(m) \cos m \theta, \quad m \in \Gamma, \quad J(m)>0 .
$$

Alternatively an interaction is specified by a translation invariant function $J$ on $\Gamma$, such that

$$
J(m)= \begin{cases}J\left(m_{i}\right) & \text { if } \exists x \in \mathbb{Z}^{d}, \quad m_{x}=m_{i} \\ 0 & \text { otherwise, }\end{cases}
$$


where $-J\left(m_{i}\right) \cos m_{i} \theta, i=1, \ldots, k$, are the $k$ observables defining the interaction. For any finite $\Lambda \subset \mathbb{Z}^{d}$ and any $\theta=\left(\theta_{\Lambda}, \theta_{\bar{\Lambda}}\right)$, (see (2.1)), the energy of $\theta_{\Lambda}$, given $\theta_{\bar{\Lambda}}$, is by definition

$$
H\left(\theta_{\Lambda} \mid \theta_{\bar{\Lambda}}\right)=-\sum_{m} J(m) \cos m \theta
$$

where the sum is over all $m$, such that the support of $m$ has a non-empty intersection with $\Lambda$. The Gibbs measure on $\Lambda$ with boundary condition $\theta$ is the probability measure $\langle\cdot\rangle_{\Lambda}^{\theta}$ on $G$ given by

$$
Z(\Lambda \mid \theta)^{-1} \exp \left(-\beta H\left(\varphi_{\Lambda} \mid \varphi_{\bar{\Lambda}}\right)\right) d \varphi_{\Lambda} \otimes \varepsilon_{\theta_{\bar{A}}}\left(d \varphi_{\bar{\Lambda}}\right) .
$$

The normalizing factor is

$$
Z(\Lambda \mid \theta)=\int d \varphi_{\Lambda} \exp \left(-\beta H\left(\varphi_{\Lambda} \mid \theta_{\bar{\Lambda}}\right)\right)
$$

and $\varepsilon_{\theta_{\bar{\Lambda}}}\left(d \varphi_{\bar{\Lambda}}\right)$ is the Dirac mass at $\theta_{\bar{\Lambda}}$ on $G(\bar{\Lambda})$. The positive parameter $\beta$ is the inverse temperature. A probability measure $\mu$ on $G$ is an equilibrium state if and only if for all finite $\Lambda$ and all observables $f$ on $G$

$$
\mu(f)=\int \mu(d \theta)\langle f\rangle_{A}^{\theta} .
$$

The free energy is

$$
f(\beta)=-\frac{1}{\beta} \lim _{\Lambda \uparrow \mathbb{Z}^{d}} \frac{1}{|\Lambda|} \log Z(\Lambda \mid \theta)
$$

which is independent of $\theta$.

\section{Correlation Inequalities}

Let $J$ be a fixed interaction given by the $k$ observables

$$
-J\left(m_{i}\right) \cos m_{i} \theta, \quad J\left(m_{i}\right)>0, \quad i=1, \ldots, k
$$

(see (2.4) and (2.5)). One of the first applications of Ginibre inequalities is to show that

$$
\left(\Lambda_{1} \subset \Lambda_{2}\right) \Rightarrow\langle\cos m \varphi\rangle_{\Lambda_{1}}^{0} \geqq\langle\cos m \varphi\rangle_{\Lambda_{2}}^{0} \geqq 0,
$$

where $\langle\cdot\rangle_{A}^{0}$ is the measure (2.7) with $\theta(x)=0$ for all $x \in \mathbb{Z}^{d}$. Using the symmetry of this measure $(\varphi \rightarrow-\varphi)$ one obtains

$$
\langle\sin m \varphi\rangle_{\Lambda}^{0}=0 .
$$

Therefore

$$
\lim _{\Lambda \uparrow \mathbb{Z}^{d}}\langle\exp \operatorname{im} \varphi\rangle_{\Lambda}^{0}=\langle\exp \operatorname{im} \varphi\rangle^{0}
$$

exists for any $m \in \Gamma$. Since this set of functions on $G$ is total among the continuous functions, the limits (3.4) define uniquely an equilibrium state $\langle\cdot\rangle^{0}$, which is translation invariant. (The last statement follows from (3.2).) Another consequence of Ginibre inequalities is the positivity

$$
\left\langle\cos m_{i} \varphi\right\rangle^{0}>0 \quad i=1, \ldots, k
$$

for the $k$ observables (3.1). 
Lemma 3.1 (Proposition 1 in [7]). For any finite $\Lambda, \theta, m$ and parameter $\alpha$

$$
\langle\cos (m \varphi-\alpha)\rangle_{\Lambda}^{\theta} \leqq\langle\cos m \varphi\rangle_{\Lambda}^{0} \text {. }
$$

Using this lemma one proves very important properties for the state $\langle\cdot\rangle^{0}$, which are summarized in the next corollary. The proof is given in [7].

Corollary 3.2. Let $\langle\cdot\rangle$ be any equilibrium state.

a) $\langle\cos m \varphi\rangle^{0} \geqq|\langle\cos m \varphi\rangle|, m \in \Gamma$,

b) if $\langle\cos m \varphi\rangle^{0}=\langle\cos m \varphi\rangle$, then $\langle\sin m \varphi\rangle=0$,

c) $\langle\cdot\rangle^{0}$ is an extremal element of the Choquet simplex of all equilibrium states.

The state $\langle\cdot\rangle^{0}$ is translation invariant and therefore it is a tangent functional to the free energy [8]. If one adds $-\lambda \cos n \theta, \lambda \geqq 0$, to the local observables (3.1), then one has a new interaction and a new free energy $f(\beta, \lambda)$ such that $f(\beta, 0)=f(\beta)$ (see (2.10)). (If $n=m_{i}$, then this is equivalent to change the coupling constants $J\left(m_{i}\right)$.)

Corollary 3.3. For any $n \in \Gamma$, one has

$$
\langle\cos n \varphi\rangle^{0}=\lim _{\lambda \downarrow 0} \frac{1}{\lambda}(f(\beta, \lambda)-f(\beta)) .
$$

Proof. This is an immediate consequence of the convexity of $f(\beta, \lambda)$ with respect to $\lambda$, and Corollary $3.2 \mathrm{a}$ ).

Let $G^{\prime}$ be a second copy of the configuration space $G$, whose elements are denoted by $\theta^{\prime}$. Let

$$
\mu=\langle\cdot\rangle^{0} \otimes\langle\cdot\rangle
$$

be the product measure on $G \times G^{\prime}$ of $\langle\cdot\rangle^{0}$ and an arbitrary equilibrium state $\langle\cdot\rangle$ on $G^{\prime}$.

Lemma 3.4 (see [5]). For any $m, n$, and $\lambda \geqq 0$

$$
\mu\left(\left(\cos m \varphi \pm \cos m \varphi^{\prime}\right) \exp \left( \pm \lambda \cos n \varphi \cos n \varphi^{\prime}\right)\right) \geqq 0 .
$$

Proof. It is sufficient to prove the lemma for the measure

$$
\langle\cdot\rangle_{A}^{0} \otimes\langle\cdot\rangle_{\Lambda}^{\theta^{\prime}}
$$

with $\Lambda$ and $\theta^{\prime}$ arbitrary. The proof is now the same as in Ginibre [6] (see also [7], where arbitrary $\theta^{\prime}$ is taken into account). Indeed, after the use of the formula

$$
2 \cos a \cos b=\cos (a+b)+\cos (a-b),
$$

and the change of variables

$$
2 \phi(x)=\varphi^{\prime}(x)+\varphi(x), \quad 2 \phi^{\prime}(x)=\varphi^{\prime}(x)-\varphi(x),
$$

one obtains the needed factorization for $\exp \left( \pm \lambda \cos n \varphi \cos n \varphi^{\prime}\right)$ and therefore the result. If one adds to the interaction the observable $h \cdot \cos p \theta(0)$, and let $h \rightarrow \infty$, then one obtains the case where the state space is $U_{p}$.

Corollary 3.5. If $\langle\cos m \varphi\rangle^{0}>0$ and $\langle\cos n \varphi\rangle^{0}=0$, then $\langle\cos (m \varphi \pm n \varphi)\rangle^{0}=0$.

Proof. Let $\mu=\langle\cdot\rangle^{0} \otimes\langle\cdot\rangle^{0}$ in Lemma 3.4. Then

$$
\mu\left(\left(\cos n \varphi+\cos n \varphi^{\prime}\right) \exp \left( \pm \lambda \cos m \varphi \cos m \varphi^{\prime}\right)\right) \geqq 0 .
$$


On the other hand

$$
\exp \left( \pm \lambda \cos m \varphi \cos m \varphi^{\prime}\right)=1 \pm \lambda \cos m \varphi \cos m \varphi^{\prime}+O\left(\lambda^{2}\right),
$$

and consequently (3.10) is equivalent to

$$
0=2\langle\cos n \varphi\rangle^{0} \geqq \pm 2 \lambda\langle\cos n \varphi \cos m \varphi\rangle^{0}\langle\cos m \varphi\rangle^{0}+O\left(\lambda^{2}\right) .
$$

Dividing by $\lambda$ and letting $\lambda \downarrow 0$, one obtains

$$
\langle\cos n \varphi \cos m \varphi\rangle^{0}\langle\cos m \varphi\rangle^{0}=0 \text {. }
$$

Using (3.8), this can be written as

$$
\langle\cos (m \varphi+n \varphi)\rangle^{0}+\langle\cos (m \varphi-n \varphi)\rangle^{0}=0 .
$$

Both terms are positive or zero by (3.2). Therefore they are zero.

Corollary 3.6. If $\langle\cos n \varphi\rangle^{0}>0$ and $\langle\cos m \varphi\rangle^{0}>0$, then $\langle\cos (m \varphi \pm n \varphi)\rangle^{0}>0$.

Proof. Let $x \in \mathbb{Z}^{d}$, and let $m_{x}$ be the translate of $m$ by $x$ :

$$
\begin{aligned}
\left\langle\cos \left(n \varphi \pm m_{x} \varphi\right)\right\rangle^{0}= & \left\langle\cos n \varphi \cos m_{x} \varphi\right\rangle^{0} \\
& \pm\left\langle\sin n \varphi \sin m_{x} \varphi\right\rangle^{0} .
\end{aligned}
$$

Let $|x| \rightarrow \infty$. Since $\langle\cdot\rangle^{0}$ is clustering

$$
\left\langle\cos \left(n \varphi \pm m_{x} \varphi\right)\right\rangle^{0} \rightarrow\langle\cos n \varphi\rangle^{0}\langle\cos m \varphi\rangle^{0}
$$

and the lemma is true for large $|x|$. If $\langle\cos (n \varphi+m \varphi)\rangle^{0}=0$, then

$$
\left\langle\cos \left(n \varphi-m_{x} \varphi\right)\right\rangle^{0}=\left\langle\cos \left(n \varphi+m \varphi-m \varphi-m_{x} \varphi\right)\right\rangle^{0}=0
$$

by Corollary 3.5 and (3.15) for $n=m$. However (3.16) and (3.15) are incompatible (for $|x|$ large). Hence $\langle\cos (n \varphi+m \varphi)\rangle^{0}>0$.

Corollary 3.7. Let $\langle\cdot\rangle$ be any equilibrium state. If $\langle\cos m \varphi\rangle^{0}=\langle\cos m \varphi\rangle$ and $\left.\langle\cos n \varphi\rangle^{0}=\langle\cos n \varphi\rangle\right\rangle 0$, then

$$
\langle\cos (n \varphi \pm m \varphi)\rangle^{0}=\langle\cos (n \varphi \pm m \varphi)\rangle .
$$

Proof. By Lemma 3.4

$$
\mu\left(\left(\cos m \varphi-\cos m \varphi^{\prime}\right) \exp \left( \pm \lambda \cos n \varphi \cos n \varphi^{\prime}\right)\right) \geqq 0 .
$$

Using (3.11) as in the proof of Corollary (3.5) one obtains

$$
\langle\cos m \varphi \cos n \varphi\rangle^{0}\langle\cos n \varphi\rangle=\langle\cos n \varphi \cos m \varphi\rangle\langle\cos n \varphi\rangle^{0},
$$

and therefore

$$
\langle\cos n \varphi \cos m \varphi\rangle^{0}=\langle\cos n \varphi \cos m \varphi\rangle .
$$

Using (3.8) one sees that

$$
\langle\cos (n \varphi \pm m \varphi)\rangle^{0}=\langle\cos (n \varphi \pm m \varphi)\rangle .
$$




\section{Translation Invariant Equilibrium States}

Let an interaction $J$ be given by $k$ local observables (3.1).

Definition. $I_{0}\left(m_{1}, \ldots, m_{k}\right)$ is the subgroup of $\Gamma$, which is generated by all $m$ in the support of $J$.

Definition. $I(\beta)$ is the set of all $m \in \Gamma$ such that $\langle\cos m \theta\rangle^{0}>0$. From the results of the last section one proves easily Proposition 4.1 .

\section{Proposition 4.1.}

a) $I(\beta)$ is a subgroup of $\Gamma$ and $I(\beta) \supseteqq I_{0}$.

b) If $\beta_{1} \geqq \beta_{2}$, then $I\left(\beta_{1}\right) \supseteqq I\left(\beta_{2}\right)$.

c) $\lim _{\beta \downarrow \beta_{0}}\langle\cos m \varphi\rangle^{0}(\beta)=\langle\cos m \varphi\rangle^{0}\left(\beta_{0}\right)$.

Proof. a) follows from Corollary 3.6 and (3.5). b) is a direct consequence of Ginibre inequalities. The last statement is proved as follows. Let $\beta>\beta_{0}$. By (3.2)

$$
\langle\cos m \varphi\rangle_{\Lambda}^{0}(\beta) \geqq\langle\cos m \varphi\rangle^{0}(\beta) \text {. }
$$

Therefore

$$
\langle\cos m \varphi\rangle_{\Lambda}^{0}\left(\beta_{0}\right) \geqq \lim _{\beta \downarrow \beta_{0}}\langle\cos m \varphi\rangle^{0}(\beta),
$$

and by taking the limit $\Lambda \uparrow \mathbb{Z}^{d}$

$$
\lim _{\beta \downarrow \beta_{0}}\langle\cos m \varphi\rangle^{0}(\beta) \leqq\langle\cos m \varphi\rangle^{0}\left(\beta_{0}\right) .
$$

Since $\langle\cos m \varphi\rangle^{0}(\beta) \geqq\langle\cos m \varphi\rangle^{0}\left(\beta_{0}\right)$, one has

$$
\lim _{\beta \downarrow \beta_{0}}\langle\cos m \varphi\rangle^{0}(\beta)=\langle\cos m \varphi\rangle^{0}\left(\beta_{0}\right) .
$$

Proposition 4.2. Let $\langle\cdot\rangle$ be any equilibrium state such that $\langle\cos m \varphi\rangle^{0}=\langle\cos m \varphi\rangle$ $>0$, for all $m \in E$, a subset of $\Gamma$. Then $\langle\cos m \varphi\rangle^{0}=\langle\cos m \varphi\rangle>0$ for all $m \in I(E)$, the subgroup of $\Gamma$ generated by $E$.

Corollary 4.3. The following statements are equivalent:

a) the free energy is differentiable at $\beta_{0}$,

b) for all translation invariant equilibrium states $\langle\cos m \varphi\rangle=\langle\cos m \varphi\rangle^{0}, m \in I_{0}$,

c) for all periodic equilibrium states $\langle\cos m \varphi\rangle=\langle\cos m \varphi\rangle^{0}, m \in I_{0}$.

Sketch of the Proof. a) is equivalent with $\left\langle\cos m_{i} \varphi\right\rangle=\left\langle\cos m_{i} \varphi\right\rangle^{0}, i=1, \ldots, k$ for all translation invariant states. Therefore, by Proposition 4.1, a) and b) are equivalent. The equivalence of $b$ ) and c) follows from Corollary $3.2 \mathrm{a}$ ).

Definition. $S_{0}$ is the annihilator of $I_{0}$, i.e. the subgroup of $G$ of all $\varphi$ such that $\exp \operatorname{im} \varphi \equiv 1$, for all $m \in I_{0} . S(\beta)$ is the annihilator of $I(\beta)$.

Since $I_{0} \subset I(\beta)$, one has $S(\beta) \subset S_{0}$. Both $I_{0}$ and $I(\beta)$ are stable under the translations of $\mathbb{Z}^{d}$, because the interaction $J$ and the state $\langle\cdot\rangle^{0}$ are translation invariant. The same property is true for $S_{0}$ and $S(\beta)$. By duality

$$
\hat{S}_{0} \cong \Gamma / I_{0}, \quad \hat{S}(\beta) \cong \Gamma / I(\beta) .
$$


Moreover the annihilator of $S(\beta)$, as subgroup of $S_{0}$, is isomorphic to $I(\beta) / I_{0}$. Therefore

$$
\left(S_{0} / S(\beta)\right)^{\wedge} \cong I(\beta) / I_{0} .
$$

There is a natural action $T_{\varphi}$ of $\varphi \in G$ on $G$ :

$$
\theta \rightarrow T_{\varphi} \theta=\theta+\varphi
$$

This action induces an action, denoted again by $T_{\varphi}$, on the probability measures on $G$ :

$$
T_{\varphi} \mu(f)=\mu\left(f \circ T_{\varphi}\right) .
$$

Using this action, $S_{0}$ is a subgroup of the symmetry group of the interaction. Indeed, for all $m \in I_{0}$ and all $\varphi \in S_{0}, \cos m \theta=\cos (m \theta+m \varphi)$. The symmetry group of the interaction is usually larger.

Example. The standard ferromagnetic Potts model with $p$ components is defined on the state space $U_{p}$. The interaction is

$$
\delta(\theta(x), \theta(y))= \begin{cases}1 & \text { if } \theta(x)=\theta(y) \\ 0 & \text { otherwise }\end{cases}
$$

where $x$ and $y$ is a pair of nearest neighbours on the lattice. Since

$$
\delta(\theta(x), \theta(y))=\frac{1}{p} \sum_{q=0}^{p-1} \cos q(\theta(x)-\theta(y)),
$$

the interaction is of the type (2.4). The group $S_{0}$ is in this case isomorphic to $\mathbb{Z}_{p}$, and hence of order $p$. On the other hand, the symmetry group is isomorphic to the group of permutations of $p$ elements.

The group $S(\beta)$ is exactly the subgroup of the symmetries of $S_{0}$, which leave the state $\langle\cdot\rangle^{0}$ invariant. Moreover all equilibrium states are $S(\beta)$-invariant by Corollary $3.2 \mathrm{a}$ ) and $\mathrm{b}$ ). The state $\langle\cdot\rangle^{0}$ has minimal symmetry. Let $\varphi \in S_{0}$ and let $S(\beta) \neq S_{0}$. The action of $\theta$ on the extremal equilibrium state $\langle\cdot\rangle^{0}$ gives a new extremal equilibrium state $\langle\cdot\rangle^{\theta}$. In particular

$$
\langle\exp \operatorname{im} \varphi\rangle^{\theta}=\exp \operatorname{im} \theta\langle\exp \operatorname{im} \varphi\rangle^{0}
$$

and the notation is justified by

$$
\langle\cdot\rangle^{\theta}=\lim _{\Lambda \uparrow \mathbb{Z}^{d}}\langle\cdot\rangle_{\Lambda}^{\theta} .
$$

Moreover $\langle\cdot\rangle^{\theta_{1}}=\langle\cdot\rangle^{\theta_{2}}$ if and only if $\left(\theta_{1}-\theta_{2}\right) \in S(\beta)$, because in (4.11) $\langle\exp \operatorname{im} \varphi\rangle^{0}>0$ for all $m \in I(\beta)$. Since all states are $S(\beta)$-invariant, there is a natural action of $S_{0} / S(\beta)$ on the Choquet simplex of all equilibrium states. The orbit of $\langle\cdot\rangle^{0}$ under the action of $S_{0} / S(\beta)$ is the set of the states $\langle\cdot\rangle^{\theta}$. For these states

$$
\langle\cos m \varphi\rangle^{\theta}=\langle\cos m \varphi\rangle^{0}, \quad \forall m \in I_{0} .
$$

Definition. $\Delta_{0}$ is the set of all equilibrium states such that $\langle\cos m \varphi\rangle^{0}=\langle\cos m \varphi\rangle$ for all $m$ in the support of $J$ (see (2.5)). 
Proposition 4.4. Let $\langle\cdot\rangle$ be any state in $\Delta_{0}$. Then there is a unique probability measure $\mu$ on $S_{0} / S(\beta)$, such that

$$
\langle\cdot\rangle=\int_{S_{0} / S(\beta)} \mu(d \theta)\langle\cdot\rangle^{\theta} .
$$

Proof. If such a representation exists, then it is unique, since all $\langle\cdot\rangle^{\theta}$ are extremal states. Let

$$
\langle\cdot\rangle=\int \lambda(d \varrho) \varrho(\cdot)
$$

be the extremal decomposition of $\langle\cdot\rangle$. Since $\langle\cdot\rangle \in \Delta_{0}$, and $I_{0}$ is a countable set, (see Proposition 4.2)

$$
\langle\cos m \varphi\rangle^{0}=\varrho(\cos m \varphi), \quad \forall m \in I_{0}
$$

$\lambda$-almost surely. In other words, $\varrho$ is in $\Delta_{0} \lambda$-almost surely. It remains to show that $\varrho=\langle\cdot\rangle^{\theta}$ for some $\theta \in S_{0} / S(\beta)$. Let $\varrho$ be an extremal equilibrium state in $\Delta_{0}$. Let

$$
\bar{\varrho}=\int_{S_{0} / S(\beta)} d \theta \varrho^{\theta},
$$

where $\varrho^{\theta}$ is obtained by the action of $\theta$ on $\varrho$ and $d \theta$ is the normalized Haar measure on $S_{0} / S(\beta)$. If $m \notin I(\beta)$ one has $\varrho(\exp \operatorname{im} \varphi)=0$. This is also true for $m \in I(\beta) \backslash I_{0}$. Indeed,

$$
\bar{\varrho}(\exp \operatorname{im} \varphi)=\int_{S_{0} / S(\beta)} d \theta \exp \operatorname{im} \theta \varrho(\exp \operatorname{im} \varphi),
$$

and by (4.6)

$$
\int_{S_{0} / S(\beta)} d \theta \exp \operatorname{im} \theta=0
$$

Therefore

$$
\bar{\varrho}(\cdot)=\int_{S_{0} / S(\beta)} d \theta\langle\cdot\rangle^{\theta},
$$

and by the uniqueness of the decomposition of $\varrho$ into extremal states, one concludes that $\varrho=\langle\cdot\rangle^{\theta}$, for some $\theta$.

Corollary 4.3 ensures that all periodic states are in $\Delta_{0}$ whenever the free energy is differentiable at $\beta$. In such a case all extremal states, which are periodic (in particular translation invariant), are in $1-1$ correspondence with the periodic elements of $S_{0} / S(\beta)$.

Definition. An inverse temperature $\beta$ is regular if the free energy is differentiable at $\beta$.

It is not difficult to prove that $\beta_{0}$ is regular if and only if $\left\langle\cos m_{i} \theta\right\rangle^{0}(\beta)$ is continuous at $\beta_{0}$ (with respect to $\beta$ ) for $i=1, \ldots, k$. Furthermore this is equivalent with $\left\langle\cos m_{i} \theta\right\rangle^{0}\left(\beta_{0}\right)=\left\langle\cos m_{i} \theta\right\rangle^{f}\left(\beta_{0}\right), i=1, \ldots, k$, where the equilibrium state $\langle\cdot\rangle^{f}$ is constructed using a free boundary condition. These statements are simple consequences of the convexity of $\beta f(\beta)$ and correlation inequalities: if $\beta_{1} \leqq \beta \leqq \beta_{2}$ and $i=1, \ldots, k$, and $\langle\cdot\rangle(\beta)$ is translation invariant,

$$
\left\langle\cos m_{i} \theta\right\rangle^{f}(\beta) \leqq\left\langle\cos m_{i} \theta\right\rangle(\beta) \leqq\left\langle\cos m_{i} \theta\right\rangle^{0}(\beta)
$$


and

$$
\begin{aligned}
& \lim _{\beta_{1} \uparrow \beta}\left\langle\cos m_{i} \theta\right\rangle^{f}\left(\beta_{1}\right)=\left\langle\cos m_{i} \theta\right\rangle^{f}(\beta), \\
& \lim _{\beta_{2} \downarrow \beta}\left\langle\cos m_{i} \theta\right\rangle^{0}\left(\beta_{2}\right)=\left\langle\cos m_{i} \theta\right\rangle^{0}(\beta) .
\end{aligned}
$$

[The proof of (4.23) is analogous to that of (4.24). See Proposition 4.1.]

Corollary 4.5. For all regular $\beta$, there is a 1-1 correspondence between the ergodic probability measures on $S_{0} / S(\beta)$ and the extremal points of the Choquet simplex of all translation invariant equilibrium states.

Remarks. 1) There are examples of ferromagnetic spin systems, for which some $\beta$ 's are not regular. In [4] p. 275 such an example is constructed. More interesting is the recent work by Kotecký and Shlosman [9] (see also Dobrushin and Shlosman [10]), where first-order phase transition in the Potts model is established. In the notations of these authors, one can choose for the states $\langle\cdot\rangle^{=}$, respectively $\langle\cdot\rangle^{\neq}$, the states $\langle\cdot\rangle^{0}$, respectively $\langle\cdot\rangle^{f}$. The first-order phase transition is established by showing that there exists a $\beta_{c}$ such that

$$
\langle\delta(\theta(x), \theta(y))\rangle^{0}\left(\beta_{c}\right)>\frac{1}{2},
$$

and in the same time

$$
\langle 1-\delta(\theta(x), \theta(y))\rangle^{f}\left(\beta_{c}\right)>\frac{1}{2} .
$$

2) The results of this section and Sect. 3 can be generalized immediately to the following situation: Let $A$ be a function defined on $\mathbb{Z}^{d}$ with values in the positive integers and which has a finite support. Let the state space be the unit ball in $\mathbb{R}^{2}$. Using polar coordinates, the state of the system at $x$ is given by $(r(x), \theta(x))$ with $0 \leqq r(x) \leqq 1$ and $\theta(x) \in T$. The $a$ priori probability measure on the state space is $v(d r) d \theta$, $v(d r) \neq \varepsilon_{0}(d r)$ the Dirac measure at 0 . The hamiltonian is formally

$$
H=-\sum_{A, m} J(A, m) r_{A} \cos m \theta
$$

with $J(A, m) \geqq 0$, and

$$
r_{A}=\prod_{x \in \operatorname{supp} A} r(x)^{A(x)} .
$$

The generalization is completely analogous to that of [4], which concerns the Ising case. In particular one has the following result: if

$$
\left\langle r_{A} \cos m \varphi\right\rangle^{0}=\left\langle r_{A} \cos m \varphi\right\rangle>0,
$$

and

$$
\left\langle r_{B} \cos n \varphi\right\rangle^{0}=\left\langle r_{B} \cos n \varphi\right\rangle>0, \quad \text { for any equilibrium state }\langle\cdot\rangle \text {, }
$$

then

$$
\left\langle r_{A} r_{B} \cos (m \pm n) \varphi\right\rangle^{0}=\left\langle r_{A} r_{B} \cos (m \pm n) \varphi\right\rangle>0 .
$$

The definitions of $I_{0}, I(\beta)$ as well as $S_{0}$ and $S(\beta)$ are the same as before. The definition of $\Delta_{0}$ is given by $\left\langle r_{A} \cos m \varphi\right\rangle^{0}=\left\langle r_{A} \cos m \varphi\right\rangle$ for all observables defining the 
interaction (together with their translates by all $x \in \mathbb{Z}^{d}$ ). The main results, Proposition 4.4 and Corollary 4.5 , are again valid. Finally one can notice that Lemma 3.4 does not require a compact state space.

3) It is possible that the same model can be described in two different ways. The Ashkin-Teller model, discussed in Sect. 5.3, provides such an example. The state space at $x$ can be realized as the group $\mathbb{Z}_{4}$ or the group $\mathbb{Z}_{2} \oplus \mathbb{Z}_{2}$. However, the crucial condition (2.4) is satisfied in both representations only if $\lambda_{1}=\lambda_{1}^{\prime} \geqq 0$ and $\lambda_{2} \geqq 0$ (see (5.10) and (5.16)). It fails in the $\mathbb{Z}_{4}$-representation for $\lambda_{1} \neq \lambda_{1}^{\prime}$. Indeed, in that representation, the hamiltonian becomes

$$
\begin{aligned}
-H= & \sum_{\langle x y\rangle}\left(\lambda_{1}+\lambda_{1}^{\prime}\right) \cos (\theta(x)-\theta(y))+\left(\lambda_{1}-\lambda_{1}^{\prime}\right) \sin (\theta(x)+\theta(y)) \\
& +\sum_{\langle x y\rangle} \lambda_{2} \cos 2(\theta(x)-\theta(y)) .
\end{aligned}
$$

The subgroup $S_{0}$ of the (internal) symmetry group is isomorphic to $\mathbb{Z}_{2}$ for $\lambda_{1} \neq \lambda_{1}^{\prime}$. However, at low temperature, there are four extremal translation invariant states, and obviously they cannot be described using this group only. In the $\mathbb{Z}_{2}$ representation of the model, condition (2.4) is valid if the coupling constants are positive. The four pure phases, mentioned above, are labelled by the elements of the group $\mathbb{Z}_{2} \oplus \mathbb{Z}_{2}$.

4) Corollary 4.5 is very simple in the case of the three-dimensional rotator model at low temperature and without magnetic field: all extremal translation invariant equilibrium states are exactly the states $\langle-\rangle^{\theta}, \theta \in T$. These states are also extremal equilibrium states (see [5]).

\section{Examples}

5.1. Introduction. In this section several ferromagnetic models are studied using correlation inequalities. The main purpose of this section is to show that it is easy to construct simple models with several phase transitions. In particular one proves the existence of two phase transitions in the Ashkin-Teller model for a suitable choice of the coupling constants. In all models there is a unique equilibrium state at small $\beta$. Therefore $S(\beta)=S_{0}$. If one increases $\beta$, one reaches a value $\beta_{1}$, where the first phase transition occurs with symmetry breakdown of the symmetry group $S_{0}$. For $\beta>\beta_{1}$, the state $\langle\cdot\rangle^{0}$ is invariant only under a subgroup $S_{1}$ of $S_{0}$. By increasing again $\beta$, one reaches a value $\beta_{2}>\beta_{1}$, where a new phase transition occurs with symmetry breakdown of $S_{1}$. For $\beta_{1}<\beta<\beta_{2}, S(\beta)=S_{1}$ and hence all equilibrium states are $S_{1}$-invariant. For $\beta>\beta_{2}$, the state $\langle\cdot\rangle^{0}$ is invariant under a subgroup $S_{2}$ of $S_{1}$ and so on. It is possible to construct models with an arbitrary number of such phase transitions. If all $\beta, \beta_{1}<\beta<\beta_{2}$, are regular, then all extremal translation invariant states (the pure phases of the model) are described by Proposition 4.4 and Corollary 4.5. If this is not the case, then there is at least one value $\beta_{*}, \beta_{1}<\beta_{*}<\beta_{2}$, where one has a first order phase transition with respect to $\beta$. However, in that particular situation, there is no symmetry breakdown of $S(\beta)$.

Convention. Since one considers only the state $\langle\cdot\rangle^{0}$ with zero boundary condition, one omits in this section the index 0 . Thus $\langle\cdot\rangle$ denotes this state when the state space is $T$, and $\langle\cdot\rangle_{p}$ denotes the same state when the state space is $U_{p}$. 
5.2. Two Lemmas. Let $J$ be an interaction given by $k$ local observables

$$
-J\left(m_{i}\right) \cos m_{i} \theta, \quad i=1, \ldots, k, \quad J\left(m_{i}\right)>0 .
$$

Lemma 5.1. Let $J$ be the interaction given by (5.1). Let $p$ and $q$ be integers such that $p=k q, k$ an integer. Then

$$
\langle\cos m \varphi\rangle_{p} \leqq\langle\cos m \varphi\rangle_{q}
$$

(where the values of $m$ are taken modulo $p$, respectively modulo $q$ ).

Proof. By Ginibre inequalities and for any finite subset $\Lambda \subset \mathbb{Z}^{d}$

$$
\langle\cos m \varphi\rangle_{p}(J) \leqq\langle\cos m \varphi\rangle_{\Lambda, p}(J) \leqq\langle\cos m \varphi\rangle_{\Lambda, p}(J, h),
$$

where on the right-hand side the interaction is given by $J$ and the local observable $-h \cos q \theta(0), h>0$. Let $h \rightarrow \infty$. Then

$$
\lim _{h \rightarrow \infty}\langle\cos m \varphi\rangle_{\Lambda, p}(J, h)=\langle\cos m \varphi\rangle_{\Lambda, q}(J) .
$$

By taking the limit $\Lambda \uparrow \mathbb{Z}^{d}$

$$
\langle\cos m \varphi\rangle_{p}(J) \leqq\langle\cos m \varphi\rangle_{q}(J) .
$$

Lemma 5.2. Let $J$ be the interaction given by (5.1). Then

a) $\langle\cos m \varphi\rangle_{p} \geqq\langle\cos m \varphi\rangle$, for any integer $p$.

Let $\left(p_{n}\right)$ be any monotone divergent sequence of integers. Then

b) $\lim _{p_{n}}\langle\cos m \varphi\rangle_{p_{n}}=\langle\cos m \varphi\rangle$.

Proof. The first part is proved as in Lemma 5.1. For any finite $\Lambda \subset \mathbb{Z}^{d}$,

$$
\lim _{p_{n}}\langle\cos m \varphi\rangle_{\Lambda, p_{n}}=\langle\cos m \varphi\rangle_{\Lambda} .
$$

Since

$$
\langle\cos m \varphi\rangle_{\Lambda, p_{n}} \geqq\langle\cos m \varphi\rangle_{p_{n}},
$$

one has

$$
\langle\cos m \varphi\rangle_{\Lambda} \geqq \varlimsup_{p_{n}}\langle\cos m \varphi\rangle_{p_{n}}
$$

Therefore

$$
\langle\cos m \varphi\rangle \geqq \varlimsup_{p_{n}}\langle\cos m \varphi\rangle_{p_{n}} .
$$

On the other hand by a)

$$
\langle\cos m \varphi\rangle \leqq \frac{\lim }{p_{n}}\langle\cos m \varphi\rangle_{p_{n}} .
$$

5.3. Ashkin-Teller Model $[11,12]$. For a special choice of the coupling constants, the Ashkin-Teller model is isomorphic to a $\mathbb{Z}_{4}$-model with hamiltonian

$$
-H=\sum_{\langle x y\rangle} \lambda_{1} \cos (\theta(x)-\theta(y))+\lambda_{2} \cos 2(\theta(x)-\theta(y)),
$$


where $\langle x y\rangle$ is a pair of nearest neighbours on the lattice $\mathbb{Z}^{d}, d \geqq 2$. Since

$$
\cos \theta(x)= \pm 1 \Leftrightarrow \sin \theta(x)=0
$$

one can introduce two Ising variables $\sigma(x)= \pm 1$ and $\tau(x)= \pm 1$, such that

$$
\begin{aligned}
& \cos \theta(x)=\frac{1}{2}(\sigma(x)+\tau(x)), \\
& \sin \theta(x)=\frac{1}{2}(\sigma(x)-\tau(x)) .
\end{aligned}
$$

Using these variables

$$
\cos 2 \theta(x)=\sigma(x) \tau(x) .
$$

The hamiltonian (5.10) becomes

$$
-H=\sum_{\langle x y\rangle}\left(\frac{\lambda_{1}}{2}(\sigma(x) \sigma(y)+\tau(x) \tau(y))+\lambda_{2} \sigma(x) \tau(y) \tau(x) \tau(y)\right),
$$

which is a symmetric case of the Ashkin-Teller hamiltonian

$$
-H=\sum_{\langle x y\rangle}\left(\frac{\lambda_{1}}{2} \sigma(x) \sigma(y)+\frac{\lambda_{1}^{\prime}}{2} \tau(x) \tau(y)+\lambda_{2} \sigma(x) \sigma(y) \tau(x) \tau(y)\right) .
$$

Without loss of generality one chooses $\lambda_{2}=1$. The symmetry group for the hamiltonian (5.16) contains four elements. If $\lambda_{1}+\lambda_{1}^{\prime}<2\left(\right.$ i.e. $\left.\frac{\lambda_{1}}{2}+\frac{\lambda_{1}^{\prime}}{2}<\lambda_{2}\right)$, then there are two phase transitions. The first one at $\beta_{1}$, which is associated with the order-parameter $\sigma(x) \tau(x)$. The second one at $\beta_{2}$, which is associated with the existence of spontaneous magnetization, $\langle\sigma(x)\rangle>0$ and $\langle\tau(x)\rangle>0$. (Here $\langle\cdot\rangle$ is the state obtained with the boundary condition $\sigma(x)=\tau(x)=1$.) Therefore, between $\beta_{1}$ and $\beta_{2}$, the system does not have spontaneous magnetization, but the variables $\tau(x)$ and $\sigma(x)$ are correlated in the sense that $\langle\sigma(x) \tau(x)\rangle>0$. If $\lambda_{1}^{\prime}>0$ and $\lambda_{1}>\lambda_{1}^{\prime}+2$, then there are again two phase transitions. The first one is characterized by a spontaneous magnetization, $\langle\sigma(x)\rangle>0$. However the two variables $\sigma(x)$ and $\tau(x)$ remain uncorrelated, $\langle\sigma(x) \tau(x)\rangle=0$ and $\langle\tau(x)\rangle=0$. At the second phase transition, $\langle\tau(x)\rangle>0$ (and of course $\langle\sigma(x) \tau(x)\rangle>0$ ). One has a similar situation for $\lambda_{1}>0$ and $\lambda_{1}^{\prime}>\lambda_{1}+2$.

The proofs are simple.

a) $\lambda_{1}+\lambda_{1}^{\prime}<2, \lambda_{2}=1$. Putting $\lambda_{1}=\lambda_{1}^{\prime}=0$ in (5.16) one obtains

$$
\langle\sigma(x) \tau(x)\rangle\left(\lambda_{1}, \lambda_{1}^{\prime}\right) \geqq\langle\sigma(x)\rangle_{I}(1),
$$

where $\langle\cdot\rangle_{I}(\mu)$ is the state, with boundary condition $\sigma(x)=+1$, of the Ising model

$$
-H=\sum_{\langle x y\rangle} \mu \sigma(x) \sigma(y) .
$$

Let $\beta(I)$ be the inverse critical temperature of the Ising model (5.18) with $\mu=1$. Then

$$
\langle\sigma(x) \tau(x)\rangle\left(\lambda_{1}, \lambda_{1}^{\prime}\right)>0 \quad \text { if } \quad \beta>\beta(I) .
$$

On the other hand

$$
\langle\sigma(x)\rangle\left(\lambda_{1}, \lambda_{1}^{\prime}\right)+\langle\tau(x)\rangle\left(\lambda_{1}, \lambda_{1}^{\prime}\right) \leqq 2\langle\sigma(x)\rangle_{I}(\tilde{\mu})
$$


where $\tilde{\mu}=\frac{\lambda_{1}}{2}+\frac{\lambda_{1}^{\prime}}{2}<1$. Inequality (5.20) is obtained by adding $-h \sum_{x} \sigma(x) \tau(x)$ in (5.16) and letting $h \rightarrow \infty$. Therefore $\langle\sigma(x)\rangle=\langle\tau(x)\rangle=0$ if $\beta\langle\beta(I) / \tilde{\mu}$.

b) $0 \leqq \lambda_{1}^{\prime}, \lambda_{1}>\lambda_{1}^{\prime}+2, \lambda_{2}=1$. Clearly

$$
\langle\sigma(x)\rangle\left(\lambda_{1}, \lambda_{1}^{\prime}\right) \geqq\langle\sigma(x)\rangle_{I}\left(\frac{\lambda_{1}}{2}\right) .
$$

Let $\langle\tau(x) \mid \sigma\rangle$ be the conditional expectation value of $\tau(x)$, given the values of $\sigma(x)$ for all $x$. This quantity is dominated by its value for $\sigma(x) \equiv 1$. Therefore

$$
\langle\tau(x)\rangle\left(\lambda_{1}, \lambda_{1}^{\prime}\right) \leqq\langle\tau(x)\rangle_{I}\left(\frac{\lambda_{1}^{\prime}}{2}+1\right) .
$$

The inequalities (5.22) and (5.23) imply the desired results.

5.4. A Rotator Model with Three Phase Transitions. The goal of this section is to study the rotator model defined by

$$
\begin{aligned}
-H= & \sum_{\langle x y\rangle} \mu_{1} \cos (\theta(x)-\theta(y)) \\
& \left.+\mu_{2} \cos 2(\theta(x)-\theta(y))+\mu_{4} \cos 4(\theta(x))-\theta(y)\right),
\end{aligned}
$$

where $\langle x y\rangle$ is a pair of nearest neighbours of $\mathbb{Z}^{d}, d \geqq 3$. For a suitable choice of the coupling constants $\mu_{1}, \mu_{2}$, and $\mu_{4}$, there are (at least) three phase transitions at $\beta_{1}, \beta_{2}$, and $\beta_{3}$. The idea is to compare this model with a $\mathbb{Z}_{4}$-model and the rotator model defined by (5.24) with $\mu_{4}=0$. The $\mathbb{Z}_{4}$-model is the symmetric case of the AshkinTeller model defined by (5.10). In the last section one has seen that this model has two phase transitions if $\lambda_{1}<\lambda_{2}$. The first one occurs at $\beta_{1}\left(4 ; \lambda_{1}, \lambda_{2}\right) \leqq \beta(I) / \lambda_{2}$ and is associated with the order-parameter $\cos 2 \theta:\langle\cos 2 \theta\rangle_{4}=0$ if $\beta<\beta_{1}$, and $\langle\cos 2 \theta\rangle_{4}>0$ if $\beta>\beta_{1}$.

The second phase transition occurs at $\beta_{2}\left(4 ; \lambda_{1}, \lambda_{2}\right) \geqq \beta(I) / \lambda_{1}$ and is associated with the order-parameter $\cos \theta$. A similar analysis holds for the rotator model (5.24) with $\mu_{1}=\lambda_{1}, \mu_{2}=\lambda_{2}, \mu_{4}=0$, if the coupling constants $\lambda_{1}$ and $\lambda_{2}$ are chosen suitably. Using Ginibre inequalities

$$
\langle\cos 2 \theta\rangle\left(\lambda_{1}, \lambda_{2}\right) \geqq\langle\cos 2 \theta\rangle\left(0, \lambda_{2}\right)=\langle\cos \theta\rangle\left(\lambda_{2}\right) .
$$

On the right hand side of (5.25) one has the rotator model (5.24) with $\mu_{1}=\lambda_{2}$, $\mu_{2}=\mu_{4}=0$. Using Lemma 5.2

$$
\langle\cos \theta\rangle\left(\lambda_{1}, \lambda_{2}\right) \leqq\langle\cos \theta\rangle_{2}\left(\lambda_{1}\right)=\langle\sigma\rangle_{I}\left(\lambda_{1}\right) .
$$

Let $\beta(R)$ be the inverse critical temperature of the rotator model with $\mu_{1}=1$, $\mu_{2}=\mu_{4}=0$. Let

$$
\beta(I) / \lambda_{1}>\beta(R) / \lambda_{2} .
$$

Inequalities (5.25), (5.26) and conditions (5.27) imply the existence of two phase transitions at $\beta_{1}\left(R ; \lambda_{1}, \lambda_{2}\right) \leqq \beta(R) / \lambda_{2}$ and $\beta_{2}\left(R ; \lambda_{1}, \lambda_{2}\right) \geqq \beta(I) / \lambda_{1}$. The first one is characterized by the order-parameter $\cos 2 \theta$ and the second one by the orderparameter $\cos \theta$. 
One now has all the necessary information to analyze the model (5.24). Without loss of generality one chooses $\mu_{2}=1, \mu_{1}=\mu_{1}^{\prime}, \mu_{4}=\mu_{4}^{\prime}$. Ginibre inequalities imply the lower bounds

and

$$
\langle\cos 4 \theta\rangle\left(\mu_{1}^{\prime}, 1, \mu_{4}^{\prime}\right) \geqq\langle\cos 2 \theta\rangle\left(1, \mu_{4}^{\prime}\right)
$$

$$
\langle\cos 2 \theta\rangle\left(\mu_{1}^{\prime}, 1, \mu_{4}^{\prime}\right) \geqq\langle\cos \theta\rangle\left(1, \mu_{4}^{\prime}\right) .
$$

On the right hand side of (5.28) and (5.29) one has the rotator model defined by (5.24), with coupling constants $\mu_{1}=1, \mu_{2}=\mu_{4}^{\prime}$, and $\mu_{4}=0$. On the other hand

$$
\langle\cos 2 \theta\rangle\left(\mu_{1}^{\prime}, 1, \mu_{4}^{\prime}\right) \leqq\langle\cos 2 \theta\rangle_{4}\left(\mu_{1}^{\prime}, 1\right)
$$

and

$$
\langle\cos \theta\rangle\left(\mu_{1}^{\prime}, 1, \mu_{4}^{\prime}\right) \leqq\langle\cos \theta\rangle_{4}\left(\mu_{1}^{\prime}, 1\right) .
$$

Since $\beta_{1}\left(4 ; \lambda_{1}, 1\right) \leqq \beta(I)$ tends to $\beta(I)$ when $\lambda_{1} \rightarrow 0$, it is possible to choose $\mu_{1}^{\prime}$ and $\mu_{4}^{\prime}$ such that

$$
\beta_{1}\left(R ; 1, \mu_{4}^{\prime}\right)<\beta_{1}\left(4 ; \mu_{1}^{\prime}, 1\right)<\beta_{2}\left(R ; 1, \mu_{4}^{\prime}\right)<\beta_{2}\left(4 ; \mu_{1}^{\prime}, 1\right) .
$$

Therefore there exist three phase transitions with symmetry breakdown at $\beta_{1} \leqq \beta_{1}\left(R ; 1, \mu_{4}^{\prime}\right)$, at $\beta_{2}$ with $\beta_{1}\left(4 ; \mu_{1}^{\prime}, 1\right) \leqq \beta_{2} \leqq \beta_{2}\left(R ; 1, \mu_{4}^{\prime}\right)$, and at $\beta_{3} \geqq \beta_{2}\left(4 ; \mu_{1}^{\prime}, 1\right)$. They are characterized, respectively, by the order-parameters $\cos 4 \theta$, $\cos 2 \theta$, and $\cos \theta$. Moreover, if $\mu_{1}^{\prime}$ decreases, then $\beta_{1}\left(4 ; \mu_{1}^{\prime}, 1\right)$ increases and if $\mu_{4}^{\prime}$ increases, then $\beta_{2}\left(R ; 1, \mu_{4}^{\prime}\right)$ decreases.

5.5. Remarks. 1. From the last example it is clear that one can construct models with $N$ phase transitions.

2. If in the last example the lattice is $\mathbb{Z}^{2}$, instead of $\mathbb{Z}^{3}$, then there is no symmetry breakdown. All equilibrium states are rotation invariant at all finite $\beta$. However, from the above analysis, there are (at least) three lines of critical points, if the coupling constants are suitably chosen. Indeed, by replacing in (5.27) $\beta(R)$ by $\beta_{c}{ }^{1}$, the critical temperature of the two-dimensional rotator model (5.24) with coupling constants $\mu_{1}=1, \mu_{2}=\mu_{4}=0$, one can prove the existence of $\beta_{1}^{\prime}>\beta_{2}^{\prime}>\beta_{3}^{\prime}$ with the following properties. Below $\beta_{k}^{\prime}, k=1,2,3,\left\langle\cos 2^{k-1}(\theta(0)-\theta(x))\right\rangle$ has an exponential decay for $|x| \rightarrow \infty$, and above $\beta_{k}^{\prime}$ the same observable has an algebraic decay.

3. Using $\mathbb{Z}_{n}$-models, for $n$ large (see [13]), on the lattice $\mathbb{Z}^{2}$, one can construct models with several lines of critical points and several phase transitions with symmetry breakdown.

4. Instead of considering ferromagnetic spin models, one can consider gauge models on a lattice. By a straightforward generalization of Sect. 5.4, one can construct models with several phase transitions associated with different Wilson loops.

5. Let $\left(\beta_{1}, \beta_{2}\right)$ be some interval of $\mathbb{R}^{+}$, in which there is exactly one phase transition with symmetry breakdown of $S(\beta)$ at $\beta_{*} \in\left(\beta_{1}, \beta_{2}\right)$. Let $S(\beta)=S_{1}$ for all $\beta_{1}<\beta<\beta_{*}$ and let $I_{1}$ be the annihilator of $S_{1}$. Therefore $\langle\cos m \varphi\rangle>0$ for $\beta_{1}<\beta<\beta_{*}$ if and only if $m \in I_{1}$. Let $n_{1} \in \Gamma$, such that $\left\langle\cos n_{1} \varphi\right\rangle=0$ for $\beta<\beta_{*}$ and $\left\langle\cos n_{1} \varphi\right\rangle>0$ for $\beta>\beta_{*}$. If $n_{2}=n_{1}+m$, with $m \in I_{1}$, then the observable $\cos n_{2} \theta$ has the same

$10<\beta_{c}<\infty$, by the recent work of Fröhlich and Spencer [13] 
property as the observable $\cos n_{1} \theta$ : its expectation value in the state $\langle\cdot\rangle$ is positive for $\beta>\beta_{*}$, but it is zero for $\beta<\beta_{*}$. We say that $\cos n_{1} \theta$ and $\cos n_{2} \theta$ are equivalent order-parameters with respect to the transition at $\beta_{*}$. This means that the possible order-parameters for the transition at $\beta_{*}$ are labelled by the elements of the quotient group $\Gamma / I_{1}$. Let $S_{2}=S(\beta) \subset S_{1}$ for $\beta>\beta_{*}$. Let $I_{2}$ be its annihilator. In this case the order-parameters associated with the transition at $\beta_{*}$ are naturally labelled by the elements of the quotient group $I_{2} / I_{1}$. Let $[\mathrm{m}]$ be an element of $I_{2} / I_{1}$, which is different from the unit element of $I_{2} / I_{1}$. Let $n \in[\mathrm{m}]$. There are two possibilities for the behaviour of the order-parameter $\cos n \theta$. Either $\langle\cos n \varphi\rangle$ is nonzero at $\beta_{*}$ and therefore discontinuous at $\beta_{*}$, or $\langle\cos n \varphi\rangle$ is zero at $\beta_{*}$ and there is a critical exponent $\alpha(n)$ describing how fast $\langle\cos n \varphi\rangle(\beta)$ goes to zero when $\beta$ tends to $\beta_{*}$. In the special case of the Ising ferromagnetic models it is possible to show that equivalent order-parameters have the same behaviour at $\beta_{*}$, [2]. In particular the critical exponents are the same. This can be generalized for all $\mathbb{Z}_{2^{k}}$-models.

Proposition 5.3. For any $\mathbb{Z}_{2^{k}}$-models, the order-parameters $\cos n_{1} \theta$ and $\cos n_{2} \theta$, for a phase transition with symmetry breakdown of $S(\beta)$ at $\beta_{*}$, have the same behaviour if they are equivalent.

Proof. To simplify the proof one considers a $\mathbb{Z}_{2^{k}}$-model with $k=3$. This means that the model is defined on the state space $U_{8}$. In that case one has

$$
2 \cos (\varphi \pm 4 \psi) \cos 4 \psi=\cos \varphi+\cos (\varphi \pm 8 \psi)=2 \cos \varphi .
$$

Equivalently

$$
\cos (\varphi-4 \psi)=\cos (\varphi+4 \psi)=\cos \varphi \cos 4 \psi .
$$

This is the key relationship. One also needs

$$
2 \cos (\varphi-\psi) \cos \psi=\cos \varphi+\cos (\varphi-2 \psi)
$$

and

$$
2 \cos (\varphi-2 \psi) \cos 2 \psi=\cos \varphi+\cos (\varphi-4 \psi) .
$$

Using the setting of Remark 5, one has to prove that $\alpha\left(n_{1}\right)=\alpha\left(n_{2}\right)$ if $n_{1}=n_{2}+m$, $m \in I_{1}$.

Since $m \in I_{1}$, one has

$$
\langle\cos m \varphi\rangle \geqq C>0, \quad \beta \in\left(\beta_{1}, \beta_{2}\right) .
$$

Using (5.33) and (5.32), and Ginibre inequaltities,

$$
\left\langle\cos \left(n_{1} \pm 4 m\right) \varphi\right\rangle \geqq\left\langle\cos n_{1} \varphi\right\rangle\langle\cos 4 m \varphi\rangle
$$

and

$$
\left\langle\cos n_{1} \varphi\right\rangle \geqq\left\langle\cos \left(n_{1} \pm 4 m\right) \varphi\right\rangle\langle\cos 4 m \varphi\rangle .
$$

Therefore $\alpha\left(n_{1}\right)=\alpha\left(n_{1} \pm 4 m\right)$. Using this result, (5.33) and (5.35), one obtains immediately that $\alpha\left(n_{1} \pm 2 m\right) \geqq \alpha\left(n_{1}\right)$. Let $\alpha\left(n_{1}, 2 m\right)$ be the critical index associated with the observable $\cos n_{1} \theta \cos 2 m \theta$. Since

$$
2 \cos n_{1} \theta \cos 2 m \theta=\cos \left(n_{1}-2 m\right) \theta+\cos \left(n_{1}+2 m\right) \theta,
$$


one has $\alpha\left(n_{1}, 2 m\right)=\alpha\left(n_{1} \pm 2 m\right) \geqq \alpha\left(n_{1}\right)$. However

$$
\left\langle\cos n_{1} \varphi \cos 2 m \varphi\right\rangle \geqq\left\langle\cos n_{1} \varphi\right\rangle\langle\cos 2 m \varphi\rangle .
$$

Therefore $\alpha\left(n_{1}, 2 m\right) \leqq \alpha\left(n_{1}\right)$ and consequently $\alpha\left(n_{1} \pm 2 m\right)=\alpha\left(n_{1}\right)$. Repeating this argument with (5.34) and using the above results, one obtains $\alpha\left(n_{1}\right)=\alpha\left(n_{1} \pm m\right)$.

6. In the same way it is possible to analyze the behaviour of generalized susceptibilities for the $\mathbb{Z}_{2^{k}}$-models (see [2]).

Acknowledgement. The author thanks J. Bricmont and J. Fröhlich for much useful discussion.

\section{References}

1. Slawny, J.: Low temperature behavior of ferromagnets. Commun. Math. Phys. 35, 297-305 (1974)

2. Lebowitz, J.L.: Coexistence of phases in Ising ferromagnets. J. Stat. Phys. 16, 463-476(1977); see also Gruber C., Lebowitz, J.L.: On the equivalence of different order parameters and coexistence of phases for Ising ferromagnet. II. Commun. Math. Phys. 59, 97 (1978)

3. Lebowitz, J.L.: Number of phases in one component ferromagnets. In: Mathematical Problems in Theoretical Physics Proceedings, Rome 1977, 68-80. Lecture Notes in Physics, Vol. 80. Berlin, Heidelberg, New York: Springer 1978

4. Bricmont, J., Lebowitz, J.L., Pfister, C.-E. : Periodic Gibbs states of ferromagnetic spin systems. J. Stat. Phys. 24, 269-277 (1981)

5. Fröhlich, J., Pfister, C.-E.: In preparation

6. Ginibre, J.: General formulation of Griffith's inequalities. Commun. Math. Phys. 16, 310-328 (1970)

7. Messager, A., Miracle-Sole, S., Pfister, C.-E.: Correlation inequalities and uniqueness of the equilibrium state for the plane rotator ferromagnetic model. Commun. Math. Phys. 58, 19-29 (1978)

8. Israel, R.B. : Convexity in the theory of lattice gases. Princeton, New Jersey: Princeton University Press 1979

9. Kotecký, R., Shlosman, S.B.: First-order phase transitions in large entropy lattice models. Commun. Math. Phys. 83, 493-515 (1982)

10. Dobrushin, R.L., Shlosman, S.B. : Phases corresponding to the local energy minima. Preprint, 1981

11. Ashkin, J., Teller, E.: Statistics of two-dimensional lattices with four components. Phys. Rev. 64, 178-184 (1943)

12. Pfister, C.-E.: Phase transitions in the Ashkin-Teller model. J. Stat. Phys. (submitted)

13. Fröhlich, J., Spencer, T.: The Kosterlitz-Thouless transition in two-dimensional abelian spin system and the Coulomb gas. Commun. Math. Phys. 81, 527-602 (1981)

Communicated by J. L. Lebowitz

Received March 25, 1982; in revised form April 19, 1982 